This item was submitted to Loughborough's Research Repository by the author.

Items in Figshare are protected by copyright, with all rights reserved, unless otherwise indicated.

\title{
The relationships between impact location and post-impact ball speed, bat torsion, and ball direction in cricket batting
}

PLEASE CITE THE PUBLISHED VERSION

https://doi.org/10.1080/02640414.2017.1389484

\section{PUBLISHER}

Taylor \& Francis

\section{VERSION}

AM (Accepted Manuscript)

\section{PUBLISHER STATEMENT}

This work is made available according to the conditions of the Creative Commons Attribution-NonCommercialNoDerivatives 4.0 International (CC BY-NC-ND 4.0) licence. Full details of this licence are available at: https://creativecommons.org/licenses/by-nc-nd/4.0/

\section{LICENCE}

CC BY-NC-ND 4.0

\section{REPOSITORY RECORD}

Peploe, Chris, Stuart A. McErlain-Naylor, Andy Harland, and Mark King. 2017. "The Relationships Between Impact Location and Post-impact Ball Speed, Bat Torsion, and Ball Direction in Cricket Batting”. figshare. https://hdl.handle.net/2134/27175. 


\title{
The relationships between impact location and post-impact ball speed, bat torsion, and ball direction in cricket batting
}

\author{
${ }^{1}$ C. Peploe, ${ }^{1}$ S.A. McErlain-Naylor, ${ }^{2}$ A.R. Harland, and ${ }^{1}$ M.A. King \\ ${ }^{1}$ School of Sport, Exercise and Health Sciences, Loughborough University, Leicestershire, LE11 3TU, UK \\ ${ }^{2}$ Sports Technology Institute, Loughborough University, Leicestershire, LE11 3QF, UK
}

\begin{abstract}
Three-dimensional kinematic data of bat and ball were recorded for 239 individual shots performed by twenty batsmen ranging from club to international standard. The impact location of the ball on the bat face was determined and assessed against the resultant instantaneous post-impact ball speed and measures of post-impact bat torsion and ball direction. Significant negative linear relationships were found between post-impact ball speed and the absolute distance of impact from the midline medio-laterally and sweetspot longitudinally. Significant cubic relationships were found between the distance of impact from the midline of the bat medio-laterally and both a measure of bat torsion and the postimpact ball direction. A 'sweet region' on the bat face was identified whereby impacts within $2 \mathrm{~cm}$ of the sweetspot in the medio-lateral direction, and $4.5 \mathrm{~cm}$ in the longitudinal direction, caused reductions in ball speed of less than $6 \%$ from the optimal value, and deviations in ball direction of less than $10^{\circ}$ from the intended target. This study provides a greater understanding of the margin for error afforded to batsmen, allowing researchers to assess shot success in more detail, and highlights the importance of players generating consistently central impact locations when hitting for optimal performance.
\end{abstract}

Keywords: velocity, sweetspot, kinematics, twist

\section{INTRODUCTION}

In cricket, the impact location of the ball on the bat face has a substantial effect on post-impact ball speed (Bower, 2012) and direction (Symes, 2006), with impacts further from the sweetspot resulting in lower ball speeds and unintended ball trajectories. Despite this, there has been very little research investigating these relationships, particularly during a dynamic hitting motion, with the majority of research instead choosing to focus on modelling impacts and their vibrational characteristics (Carré et al., 2004; Brooks et al., 2006).

Only two studies have attempted to quantify the relationships between impact location and post-impact ball speed and direction in cricket. Bower (2012) used a pendulum to swing a cricket bat at low speeds $\left(5.8-7.3 \mathrm{~ms}^{-1}\right.$ compared to $18.9-$ $20.8 \mathrm{~ms}^{-1}$ in a realistic hitting action; Peploe et al., 2014) towards a stationary suspended cricket ball. Impacts occurring centrally and $1 \mathrm{~cm}$ either side of the midline were found to generate significantly higher post-impact ball speeds and apparent coefficients of restitution (ACOR) than impacts occurring 2 and $3 \mathrm{~cm}$ from the centre. The same trend was found in the longitudinal direction, with impacts occurring $15-20 \mathrm{~cm}$ from the toe of the bat generating significantly higher post-impact ball speeds than impacts either higher or lower on the bat face.

In a more detailed study, Symes (2006) assessed the relationships between impact location and post-impact ball speed, bat torsion, and ball direction in a range of bat designs. In the experimental protocol, a ball launcher (Justham \& West, 2009) was used to project a cricket ball horizontally into impact with a freely suspended cricket bat. An optimal impact location was found in the longitudinal direction of approximately $17 \mathrm{~cm}$ from the toe of the bat, with impacts further towards the handle and toe of the bat resulting in a linear reduction in ACOR. A similar study in tennis 
(Elliott et al., 1980) found ball speed to decrease with impacts further in the mediolateral and longitudinal directions from a point along the midline of the racket, also identifying an optimal impact area to generate maximum ball rebound speed. Results for off-centre impacts in cricket (Symes, 2006) displayed a series of linear relationships between the change in bat angle, the rate of bat polar rotation, and the distance of impact from the midline, finding additional bat rotation to occur following impacts further from the midline. This resulted in a further linear relationship, with the ball rebounding further from the intended target as impacts occurred further from the midline. Likewise, Knudson (1993) found off-centre impacts to project the ball further from a line normal to the racket face when compared to central impacts in tennis.

Although previous studies have identified relationships between impact location and shot outcome, their application to dynamic hitting actions is limited, and the margins for error in terms of impact location afforded to a player have not been discussed. The low relative velocity between bat/racket and ball (Elliott et al., 1980; Symes, 2006; Bower, 2012) differs from a realistic impact and neglects bat/racket speed; one of the key factors for determining bat performance (Smith, 2001; Nathan, 2003). Furthermore, the grip interface has been shown to affect post-impact ball velocity in tennis. A firmer grip was found to cause a significant increase in postimpact ball velocity for off-centre impacts, and a statistically insignificant increase in reaction impulse (17\%) and post-impact ball velocity (7\%) for central impacts (Elliott, 1982). Although no relationship has been reported between grip firmness and postimpact ball velocity for wooden baseball bats (Weyrich et al., 1989) it is possible that the clamped (Bower, 2012) and freely suspended (Symes, 2006) grip conditions may influence shot outcome when compared to a human grip, particularly during offcentre impacts. Players in a number of sports (Mclntyre \& Pfautsch, 1982; Noble \& Eggeman, 1982; Shibayama \& Ebashi, 1983; Stretch et al., 1995) have also been shown to reduce their grip forces prior to impact, further limiting the application of a clamped grip interface. In addition, the fixed nature of the bat during the pendulum test (Bower, 2012) does not allow investigation of post-impact bat torsion and ball direction.

Attempting to accurately identify the impact location of a ball on the bat face during a dynamic hitting action in a realistic cricket environment has presented a problem for researchers. The high ball speeds (release speeds between 32.0 and $40.0 \mathrm{~ms}^{-1}$; Worthington et al., 2013) and short impact durations (1.0 - $1.5 \mathrm{~ms}$; Symes, 2006) typically found in cricket, cause substantial difficulties in identifying the time of impact and therefore its location on the bat face. The advent of a novel curve fitting methodology to determine the impact location of a cricket ball on a bat face from three-dimensional marker data, allows researchers scope to investigate the relationships between impact location and post-impact bat and ball kinematics during a dynamic hitting action. This methodology (Peploe et al., 2017) has been found to accurately establish impact location to within $6.4 \pm 4.2$ and $7.1 \pm 4.4 \mathrm{~mm}$ in the medio-lateral and longitudinal directions of the bat face respectively, while also estimating impact timing and instantaneous post-impact ball speed and direction. As such, the aim of this study was to assess the effects of medio-lateral and longitudinal bat-ball impact location on post-impact ball speed, and in the medio-lateral direction on peak change in rate of bat polar rotation and on ball direction, during a dynamic hitting task. This will provide a more detailed understanding of the margin for error afforded to cricket batsmen in terms of impact location during a dynamic hitting motion. 


\section{METHODS}

All testing was conducted at the England \& Wales Cricket Board National Cricket Performance Centre in Loughborough, UK, on a standard sized artificial cricket pitch. Data was recorded using an 18 camera Vicon Motion Analysis System (OMG Plc, Oxford, UK) operating at $250 \mathrm{~Hz}$. Testing procedures were explained to each participant involved, and informed written consent was obtained in accordance with the guidelines of the Loughborough University Ethical Advisory Committee. All participants completed a thorough self-selected warm-up before data collection.

\section{Data Collection}

Twenty experienced male cricketers $(23 \pm 3$ years, $1.82 \pm 0.04 \mathrm{~m}, 80.0 \pm 7.8 \mathrm{~kg})$ participated in this investigation. Participants ranged from club to international batsmen, thus a range of impact locations and ball velocities were generated. Five $15 \times 15 \mathrm{~mm}$ squares of $3 \mathrm{M}$ reflective scotch tape were attached to a standard size adult cricket ball (Figure 1). Four $14 \mathrm{~mm}$ spherical reflective markers were positioned on the back corners of the blade of each participant's cricket bat (Figure 2). Participants performed a series of hitting trials (14 \pm 4$)$ against a bowling machine (BOLA Professional), each time attempting to hit a forward drive for maximal distance straight down the ground. Ball resultant inbound speed on the approach to impact, calculated using differentiated ball position data over a 40 ms interval, was $25.0 \pm 1.3$ $\mathrm{ms}^{-1}$. Only trials where the ball was projected in a forward direction after impact were selected for analysis, leaving a total of 239 trials.
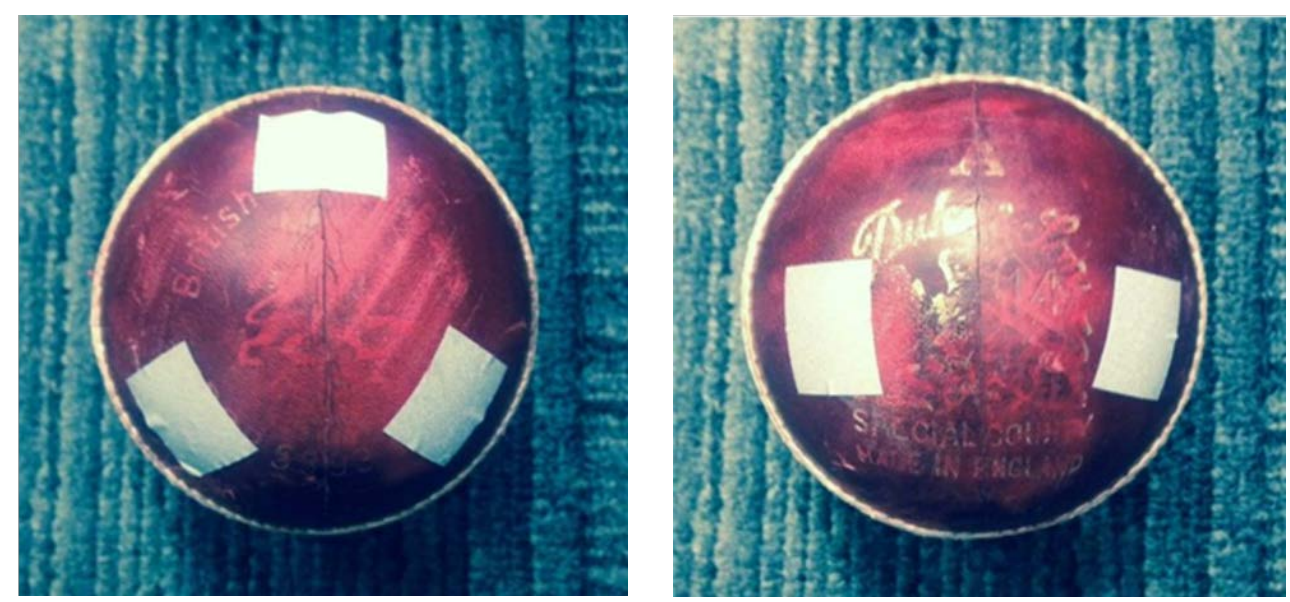

Figure 1. Reflective tape positioned on the cricket ball.

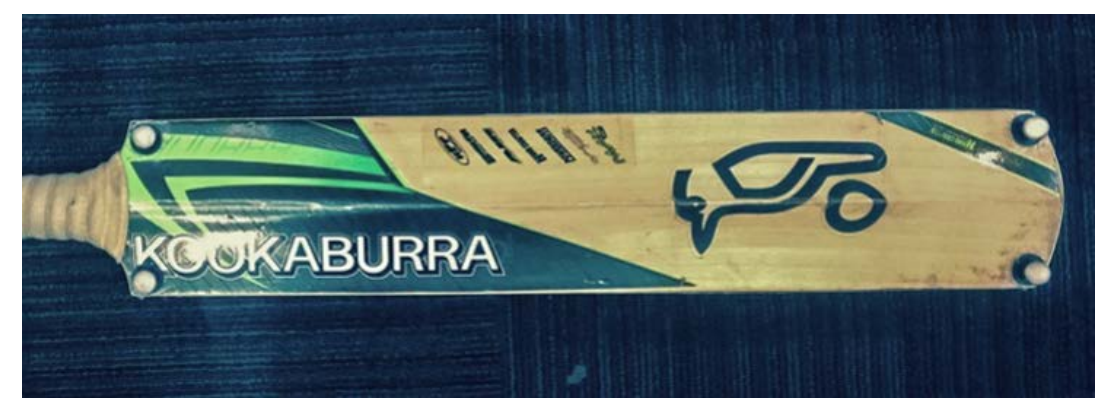

Figure 2. Reflective markers positioned on the blade of the cricket bat. 


\section{Data Reduction}

Bat and ball marker position data were manually labelled and processed for each trial. The single ball displacement equation methodology of Peploe et al. (2017) was then used to determine the impact location of the ball on the bat face (accurate to within $7.4 \pm 4.8 \mathrm{~mm}$ medio-laterally and $9.2 \pm 4.3 \mathrm{~mm}$ longitudinally of measured locations). Curves were fitted separately to the pre- and post-impact phases of the unfiltered ball position data against time in three axes according to logarithmic equations determined from mechanical principles. Separate Fourier series models were similarly fitted to the four corners of the bat face against time during the downswing prior to ball impact. Time of impact was determined based upon the intersection of pre- and post-impact curves. Impact location was calculated from ball and bat face curves at this time, and resultant instantaneous post-impact ball speed was determined via differentiation of the post-impact ball curves.

\section{Data Analysis}

Initially, the goodness of fit ( $\mathrm{R}^{2}$ and RMSE) of each curve, and the difference in estimated impact time between curves in the vertical and anterior-posterior planes, were established and assessed as measures of accuracy within the methodology. The resultant instantaneous post-impact ball speed, calculated from the curve equations, was also compared to ball speed calculated via differentiation of ball position over a $40 \mathrm{~ms}$ interval.

The calculated impact locations in the medio-lateral and longitudinal directions of the bat face were then assessed against the resultant instantaneous post-impact ball speed. Firstly, the distances of impact from a virtual marker on the bottom corner of the bat face medio-laterally and longitudinally were plotted against resultant post-impact ball speed, and the relationships assessed. A virtual sweetspot position was subsequently defined based on the work of Bower (2012), as being located on the midline of the bat in the medio-lateral direction, and $17.5 \mathrm{~cm}$ from the toe of the bat in the longitudinal direction. The relationships between the absolute distance of impact from the sweetspot in both directions individually, and the resultant postimpact ball speed were assessed. Finally, a forwards stepwise linear regression was used to predict the post-impact ball speed from the impact location in the mediolateral and/or longitudinal directions combined.

Peak post-impact rate of change in bat polar rotation about its longitudinal axis (subsequently referred to as a measure of bat torsion), and the post-impact ball direction relative to a line perpendicular to the bat face at the time of impact were then calculated for each trial. These were individually compared to the calculated impact location in the medio-lateral direction. The effects of impact location in the longitudinal direction on bat torsion and ball direction were assumed to be negligible (King et al., 2012) and so were not assessed. Initially the rate of bat polar rotation (about the midline of the bat face) was calculated from 3D bat marker positions, and the absolute peak between impact (timing determined using Peploe et al., 2017) and $0.02 \mathrm{~s}$ after impact was defined. A measure of post-impact bat torsion was then determined as the difference between the peak measured rate of bat polar rotation, and the mean pre-impact rate of bat polar rotation during a similar period prior to impact. Both the angle of the bat face and post-impact ball direction were calculated relative to the global coordinate system, from bat displacement curves at the time of impact, and the post-impact ball displacement curves respectively (Peploe et al., 2017). Finally, the relationship between peak change in the rate of bat polar rotation and post-impact ball direction was assessed. 
All statistical analyses were performed within SPSS v.23 (IBM Corporation, Armonk, NY, USA). Pearson product moment correlations were used to establish relationships, with 1000 bootstrap replication 95\% confidence intervals determined for all correlations, and a P-value $<0.05$ indicating statistical significance. Linear regressions were used to predict post-impact ball speed from impact location on the bat in both directions separately before a forwards stepwise linear regression (parameters included at $P<0.05$ ) predicted post-impact ball speed from the mediolateral and/or longitudinal impact locations combined. Similarly, regression models would be rejected if coefficient $95 \%$ confidence intervals included zero or if correlations, tolerance statistics or variance inflation factors showed any evidence of multicollinearity (Bowerman \& O'Connell, 1990; Draper \& Smith, 1998; Field, 2013; Menard, 1995; Myers, 1990). To confirm the normality of the standardised residuals in the regression models, Shapiro-Wilk tests for normality were performed. The Pvalues ranged from 0.18 to 0.49 , indicating no evidence against the assumption of normality of the residuals. The percentage of variance in the dependent variable (post-impact ball speed) explained by the independent variable(s) in the stepwise regression was determined by Wherry's (1931) adjusted $\mathrm{R}^{2}$-value. This represents an attempt to estimate the proportion of variance that would be explained by the model had it been derived from the entire population from which the sample was taken.

\section{RESULTS}

$\mathrm{R}^{2}$ and RMSE values for the goodness of fit of the pre- and post-impact ball curves averaged $0.99 \pm 0.04$ and $9.8 \pm 4.3 \mathrm{~mm}$, while the $R^{2}$ and RMSE values for the bat curves averaged $0.99 \pm 0.03$ and $1.0 \pm 0.9 \mathrm{~mm}$ respectively (Table 1 ).

Table 1. Goodness of fit statistics for ball and bat curves (mean \pm SD).

\begin{tabular}{lcl}
\hline & \multicolumn{1}{c}{$\mathrm{R}^{2}$} & \multicolumn{1}{c}{$\mathrm{RMSE}(\mathrm{mm})$} \\
\hline Ball medio-lateral post & $0.97 \pm 0.07$ & $10.3 \pm 4.6$ \\
Ball anterior-posterior pre & $1.00 \pm 0.00$ & $10.6 \pm 4.2$ \\
Ball anterior-posterior post & $0.99 \pm 0.03$ & $11.4 \pm 5.0$ \\
Ball vertical pre & $0.99 \pm 0.03$ & $8.4 \pm 2.8$ \\
Ball vertical post & $1.00 \pm 0.02$ & $8.2 \pm 3.2$ \\
Bat medio-lateral pre & $0.98 \pm 0.06$ & $1.0 \pm 0.9$ \\
Bat anterior-posterior pre & $1.00 \pm 0.00$ & $1.1 \pm 1.1$ \\
Bat vertical pre & $1.00 \pm 0.01$ & $0.8 \pm 0.8$ \\
\hline
\end{tabular}

Note: pre/post relates to pre-/post-impact phase curves

Assessment of the estimated impact time between curves in the vertical and anterior-posterior planes showed a mean difference of $1.3 \pm 1.0 \mathrm{~ms}$. Instantaneous post-impact ball speed for the 239 trials was $28.1 \pm 4.2 \mathrm{~ms}^{-1}$ (range $17.8-39.5 \mathrm{~ms}^{-1}$ ). Comparison with ball speed calculated via differentiation of ball position data, again over a $40 \mathrm{~ms}$ interval, displayed an absolute difference of $0.6 \pm 0.6 \mathrm{~ms}^{-1}$.

Significant negative quadratic relationships against resultant post-impact ball speed were observed (Figure 3) for the distance between the ball centre and a virtual marker on the bottom corner of the bat face at the time of impact, both medio-laterally $\left(R^{2}=0.289 ; p<0.001\right)$ and longitudinally $\left(R^{2}=0.241, p<0.001\right)$. This indicates the 
presence of an optimal impact location in both directions for generating high postimpact ball speed. Correlations revealed significant negative relationships between the absolute distance of the ball centre from the midline $(r=-0.53 ; 95 \% \mathrm{Cl}=-0.61$, $0.44 ; p<0.001)$ in the medio-lateral direction, and sweetspot $(r=-0.48 ; 95 \% \mathrm{Cl}=-$ $0.57,-0.38 ; p<0.001)$ in the longitudinal direction, at the time of impact, and postimpact ball speed (Figure 4). Linear regression analysis of these relationships allowed predictive equations for post-impact ball speed to be formed in the mediolateral (Eq. 1; $\mathrm{R}^{2}=0.28$; $\left.\mathrm{SEE}=3.54 \mathrm{~ms}^{-1}\right)$ and longitudinal $\left(\mathrm{Eq} .2 ; \mathrm{R}^{2}=0.23\right.$; $\mathrm{SEE}=$ $3.65 \mathrm{~ms}^{-1}$ ) directions. Confidence intervals (95\%) for the absolute impact location coefficients were -127.3 to -83.8 medio-laterally, and -57.6 to -35.8 longitudinally. Stepwise linear regression enabled the determination of Eq. 3, explaining $48.4 \%$ of observed variation in post-impact ball speed from impact location, with medio-lateral and longitudinal impact locations on the bat face both included in the final regression model $\left(p<0.001\right.$; SEE $\left.=2.99 \mathrm{~ms}^{-1}\right)$. Confidence intervals $(95 \%)$ for the coefficients were -120.0 to -83.2 medio-laterally, and -53.4 to -35.7 longitudinally.

$$
\begin{aligned}
& v=-105.56 y+31.34 \\
& v=-46.68 y+30.71 \\
& v=-101.57 x-44.54 y+33.70
\end{aligned}
$$

where $\mathrm{v}=$ post-impact ball speed $\left(\mathrm{ms}^{-1}\right) ; x=$ absolute medio-lateral distance of impact from the sweetspot $(\mathrm{m})$; and $\mathrm{y}=$ absolute longitudinal distance of impact from the sweetspot $(\mathrm{m})$.
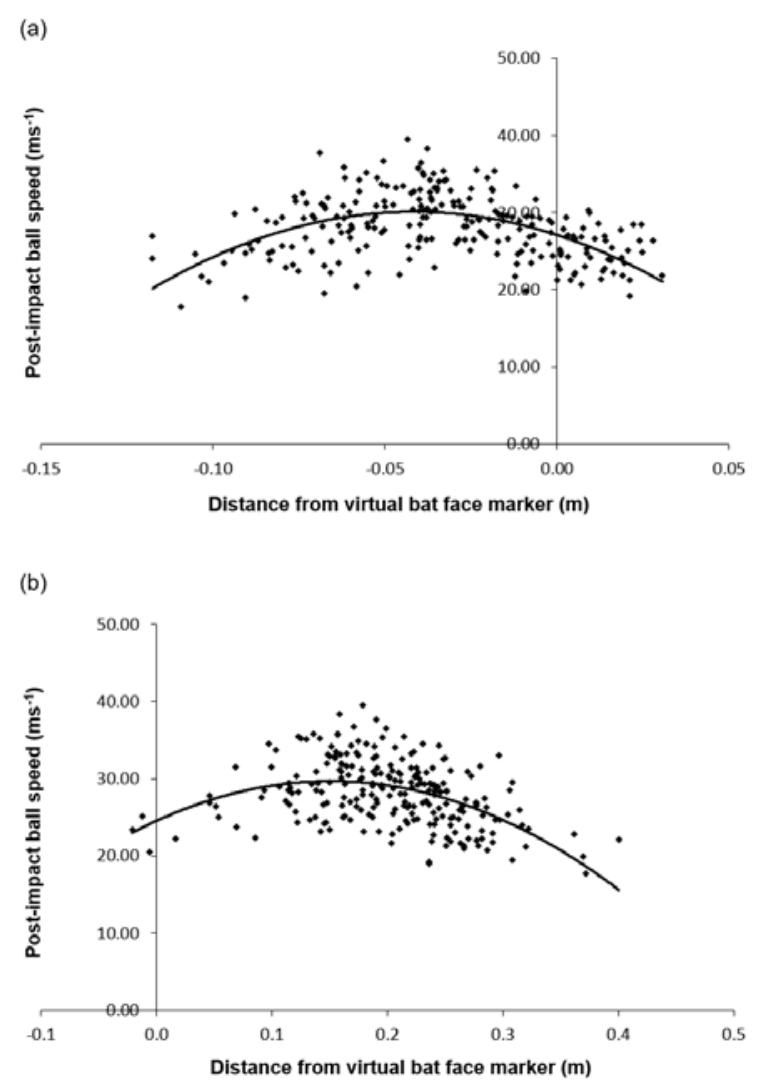

Figure 3. The distance between the ball centre and a virtual marker on the bottom corner of the bat face at the time of impact in the medio-lateral (a) and longitudinal (b) directions against resultant post-impact ball speed. 

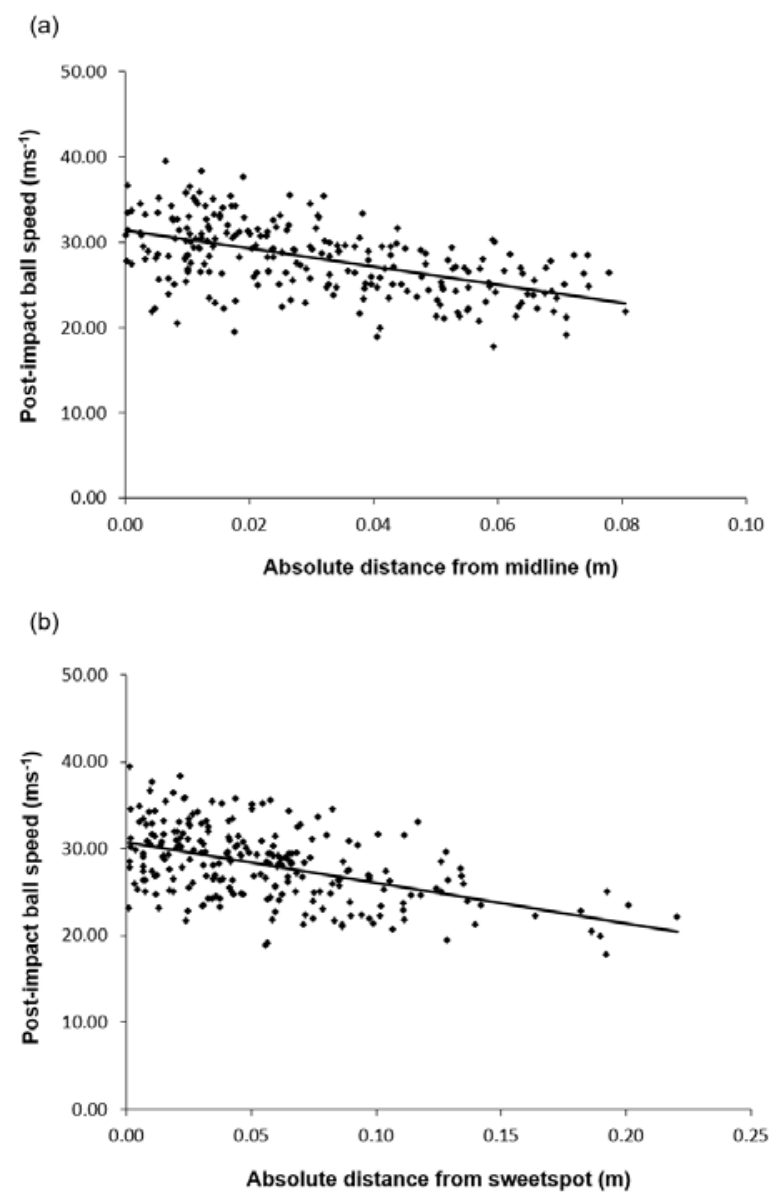

Figure 4. The absolute distance between the ball centre and the midline (medio-lateral; a) and sweetspot (longitudinal; b) of the bat face at the time of impact against resultant postimpact ball speed.

The medio-lateral distance of impact from the midline of the bat and the previously defined measure of bat torsion displayed a significant cubic fit $\left(\mathrm{R}^{2}=0.89\right.$; $\mathrm{p}<0.001$; Figure 5). Analysis of this cubic relationship also allowed a predictive equation to be formed for the peak change in the rate of bat polar rotation according to the medio-lateral distance of impact from the midline of the bat (Eq. 4).

$$
\Delta t=-33291569 x^{3}+686849 x^{2}+264594 x-1532
$$

where $\Delta t=$ change in the rate of bat polar rotation $\left({ }^{\circ} \cdot \mathrm{s}^{-1}\right)$; and $x=$ absolute medio-lateral distance of impact from the midline $(\mathrm{m})$. 


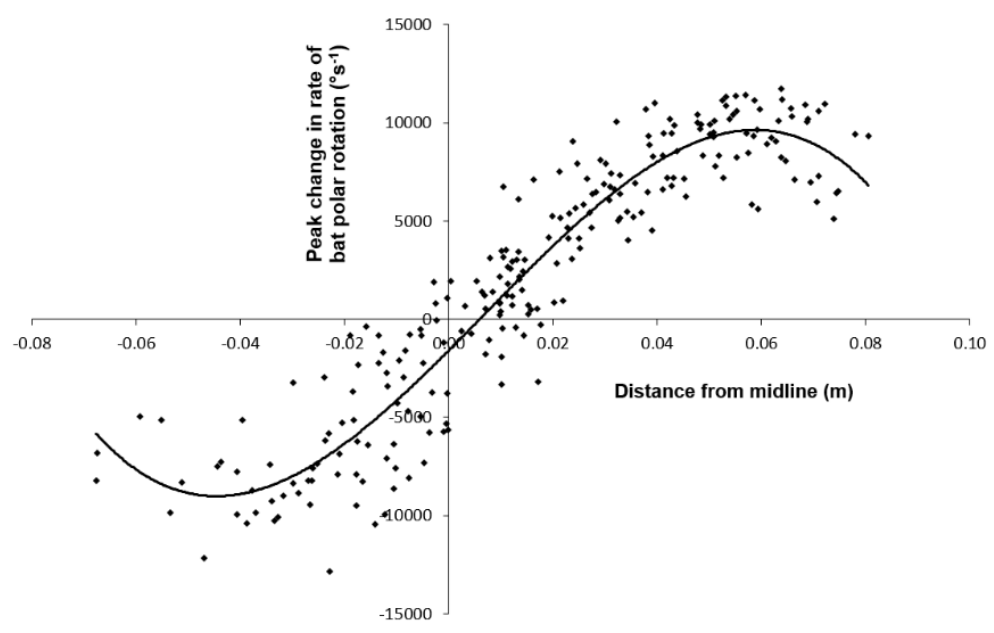

Figure 5. The distance of impact from the midline of the bat (medio-laterally) against the peak change in the rate of bat polar rotation as a result of impact.

Comparison of the difference between the post-impact ball direction and the angle of the bat face immediately prior to impact with the medio-lateral impact location revealed a significant cubic relationship $\left(R^{2}=0.70 ; p<0.001\right.$; Figure 6). Analysis of this cubic relationship also allowed a predictive equation to be formed for the deviation of the ball trajectory from a line perpendicular to the bat face at the time of impact, according to the medio-lateral distance of impact from the midline of the bat (Eq. 5). This post-impact ball direction was also shown to correlate significantly with the previously defined measure of bat torsion $(r=0.73 ; 95 \% \mathrm{Cl}=0.68,0.78 ; p<$ $0.001)$.

$$
d=60543 x^{3}-681 x^{2}+494 x-1.5
$$

where $d=$ deviation of ball trajectory from a line perpendicular to the bat face at the time of impact $\left({ }^{\circ}\right)$; and $x=$ absolute medio-lateral distance of impact from the midline $(\mathrm{m})$.

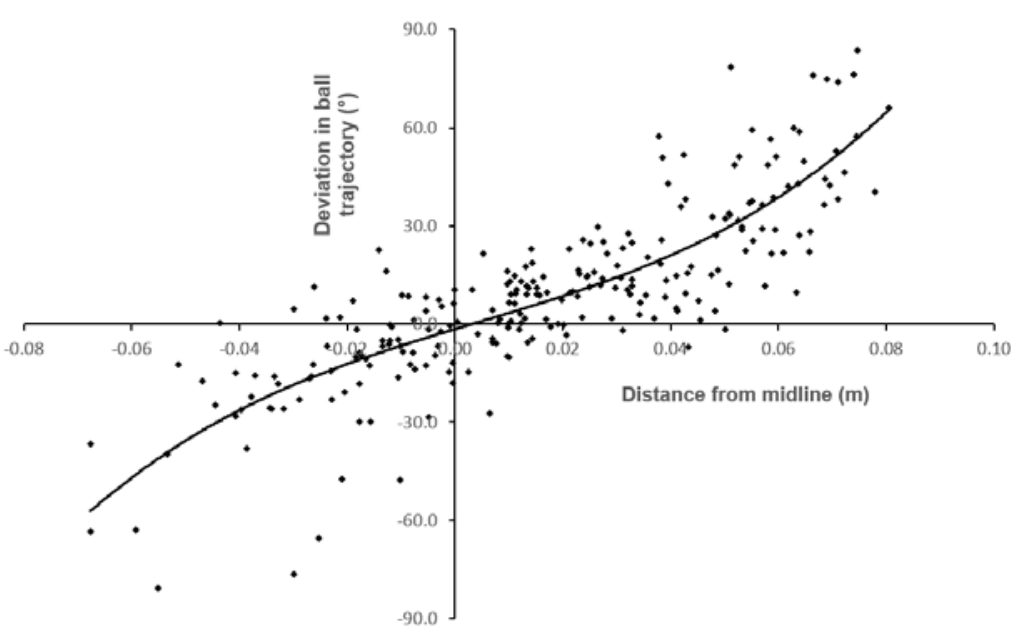

Figure 6. The distance of impact from the midline of the bat (medio-laterally) against the deviation in post-impact ball trajectory from a line perpendicular to the bat face immediately prior to impact. 
Finally, the regression equations (Eq. 3 and 5) for post-impact ball speed and ball direction allowed visual representations of the effects of impact location on the two primary outcome variables to be created (Figure 7). The combined effects of medio-lateral and longitudinal impact locations are displayed as areas causing incremental reductions in post-impact ball speed (Figure 7a), showing that impacts within $2 \mathrm{~cm}$ of the sweetspot in the medio-lateral direction, and $4.5 \mathrm{~cm}$ in the longitudinal direction, cause reductions in ball speed of less than $6 \%$. The effects of medio-lateral impact location on absolute ball direction values are also displayed (Figure $7 \mathrm{~b}$ ), showing that impacts within $2 \mathrm{~cm}$ of the midline cause deviations in ball direction of less than $10^{\circ}$ from the intended target.

(a)

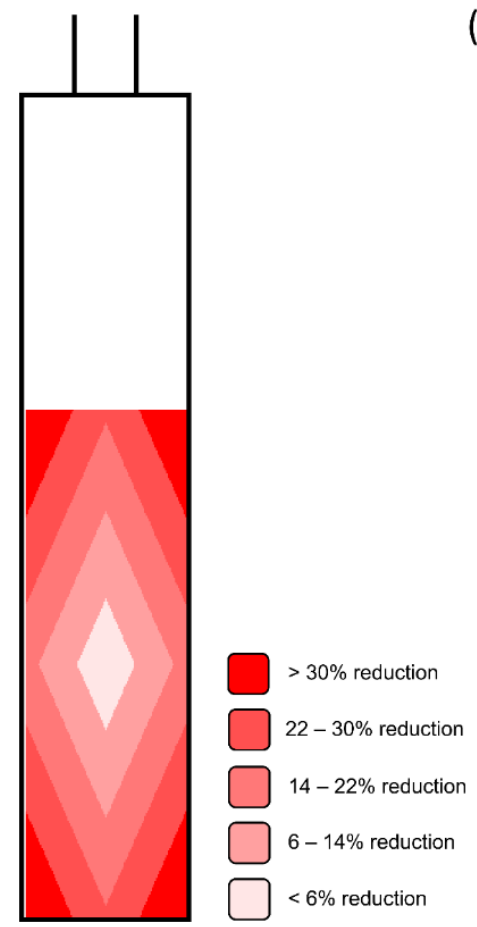

(b)

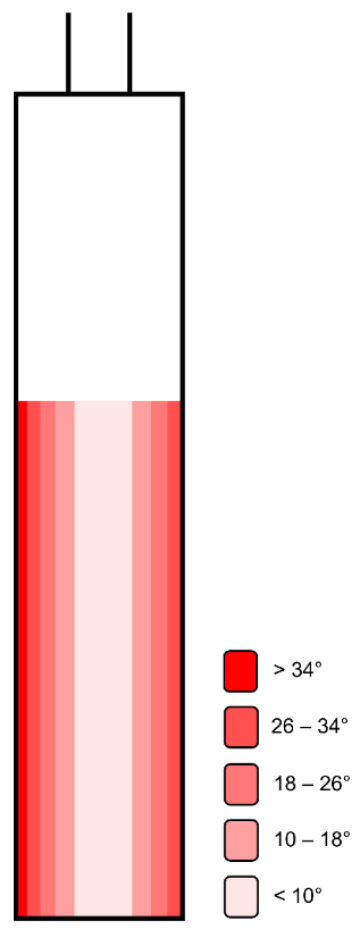

Figure 7. Visual representations of the effect of impact location on post-impact ball speed (a), and ball direction (b).

\section{DISCUSSION}

An investigation into the effects of impact location on post-impact ball speed, peak change in rate of bat polar rotation, and ball direction in cricket batting has been presented. Relationships between impact location and each outcome variable have been assessed, and the margin for error afforded to batsmen in terms of impact location has been determined. This has allowed a 'sweet region' on the bat to be defined, whereby impacts within $2 \mathrm{~cm}$ in the medio-lateral direction, and $4.5 \mathrm{~cm}$ in the longitudinal direction, cause reductions in ball speed of less than $6 \%$ from the optimal value, and deviations in ball direction of less than $10^{\circ}$ from the intended target.

The mean $\mathrm{R}^{2}$ and RMSE values for pre- and post-impact ball curves $(0.99 \pm$ 0.04 and $9.8 \pm 4.3 \mathrm{~mm})$, and pre-impact bat curves $(0.99 \pm 0.03$ and $1.0 \pm 0.9 \mathrm{~mm}$, ) were found to be similar to those achieved during the validation of the impact location methodology (Peploe et al., 2017). This indicates a similar quality of curve fitting, and thus comparable errors in terms of impact location. The difference in estimated impact timing between the anterior-posterior and vertical planes was $1.3 \pm 1.0 \mathrm{~ms}$, 
and when compared to differentiation over a $40 \mathrm{~ms}$ interval, the resultant ball speed obtained via curve fitting revealed an absolute difference of $0.6 \pm 0.6 \mathrm{~ms}^{-1}$. The proximity of these two sets of results to each other, and their similarity to findings achieved during validation of the methodology (Peploe et al., 2017), further supports the suitability of this data for impact location analysis and for a series of valid conclusions to be drawn.

The significant quadratic relationships displayed in Figure 3 indicate the presence of an optimal impact location, or sweetspot, in both the medio-lateral and longitudinal directions on the bat face, where impact further from that point generates a lower resultant ball speed. This is confirmed by the significant linear correlations between the absolute distances of impact from the previously defined sweetspot in both the medio-lateral and longitudinal directions against resultant post-impact ball speed (Figure 4). This finding agrees with the work of Symes (2006), who found a linear decrease in ACOR with impacts further from the sweetspot longitudinally. The fact that the peak measured post-impact ball speed occurred at the virtual sweetspot in the longitudinal direction, supports the work of Bower (2012) and Symes (2006), in finding the highest rebound speeds to occur during impacts approximately $17.5 \mathrm{~cm}$ from the toe of the bat.

The stepwise linear regression utilising the impact location on the bat in both directions explained a greater proportion of the variation, with a lower standard error of the estimate (adjusted $\mathrm{R}^{2}=0.48$; SEE $=2.99 \mathrm{~ms}^{-1}$ ), than either single parameter regression alone (adjusted $R^{2}=0.28,0.23$; SEE $=3.54 \mathrm{~ms}^{-1}, 3.65 \mathrm{~ms}^{-1}$ ), highlighting that it is a combination of the distance of impact from the sweetspot both mediolaterally and longitudinally that truly affects the post-impact ball speed. Although batball impact location and post-impact ball speed have been shown to be strongly correlated, many other factors such as pre-impact bat and ball speed are also likely to have an effect; this can be seen as accounting for the imperfect relationships displayed here. Investigation of the regression equation for this relationship revealed that impacts occurring $2 \mathrm{~cm}$ from the midline of the bat in the medio-lateral direction, or $4.5 \mathrm{~cm}$ from the sweetspot in the longitudinal direction, caused a reduction in postimpact ball speed of $6.0 \%(95 \% \mathrm{Cl}: 4.8-7.1 \%)$ in comparison to an impact directly on the sweetspot.

Comparison of the medio-lateral distance of impact from the midline of the bat with the measure of bat torsion showed a significant cubic relationship $\left(R^{2}=0.89, p<\right.$ 0.001 ; Figure 5) in line with the hypothesis. This indicates that for impacts occurring across the majority of the bat face, an increased distance of impact from the midline of the bat generates a greater peak change in the rate of bat polar rotation (as in the study by Symes; 2006), while impacts occurring near the edge of the bat generate less torsion due to more of an oblique impact. Although the study performed by Symes (2006) states a predominantly linear relationship between impact location and the rate of bat polar rotation, there is some evidence of a reduction in bat torsion with impacts towards the edge of the bat. It is also possible that the non-normal nature of impacts in this study, and the increased momentum created during impact by the fast-moving bat, are the cause of the comparatively marked decrease in bat torsion during impacts towards the edge of the bat face. This suggests that, while future studies may be able to assess post-impact bat torsion as a measure of success in cricket batting, researchers should take care when interpreting impacts occurring near the edge of the bat, as the torsion magnitude alone is likely to be misleading.

Finally, the cubic relationship $\left(R^{2}=0.70, p<0.001\right.$; Figure 6$)$ found when comparing the difference between the post-impact ball direction and the angle of the 
bat face immediately prior to impact with the medio-lateral impact location on the bat, indicates a similar trend as that previously discussed between impact location and bat torsion. Impacts occurring further medio-laterally from the midline of the bat caused the ball to depart on an unwanted trajectory away from a line perpendicular to the bat face, likely due to increased bat torsion. Indeed, there was a significant positive correlation between the bat torsion measure and post-impact ball direction ( $r$ $=0.73 ; 95 \% \mathrm{Cl}=0.68,0.78 ; \mathrm{p}<0.001$ ). However, impacts occurring towards the edge of the bat displayed some additional deviation in ball trajectory, most likely due to the oblique nature of impact in these situations causing the ball to slide off the bat face with reduced bat torsion. The medio-lateral convex curvature of a typical bat face may also further contribute to the oblique nature of impacts towards the edge of the bat. Investigation of the predictive equations (Eq. 4 and 5) for bat torsion and post-impact ball direction revealed that impacts $2 \mathrm{~cm}$ away from the midline of the bat in the medio-lateral direction generated a peak change in the rate of bat polar rotation of $3800{ }^{\circ} \mathrm{s}^{-1}$, causing the ball to depart up to approximately $9^{\circ}$ away from the intended trajectory.

\section{CONCLUSIONS}

Investigation of the effects of impact location on resulting post-impact bat and ball motion has shown batsmen to possess a relatively small margin for error in terms of impact location in order to generate their intended shot outcome. Impacts away from the sweetspot of the bat have been found to cause a substantial reduction in post-impact ball speed, and a decrease in shot accuracy towards the intended target. This study therefore provides a greater understanding of the relationships between impact location and post-impact ball speed and direction, as well as the margin for error afforded to a batsman when hitting a cricket ball. This highlights the importance of players generating consistently central impact locations when hitting, and will allow researchers to assess shot outcome and impact characteristics in more detail during future studies.

\section{ACKNOWLEDGEMENTS}

This project was part-funded by the England and Wales Cricket Board (ECB). 


\section{REFERENCES}

Bower, R. (2012). The sweet spot of a cricket bat for low speed impacts. Sports Engineering, 15(2), 53-60.

Bowerman, B. L., \& O'Connell, R. T. (1990). Linear statistical models: An applied approach (2nd ed.). Belmont, CA: Duxbury.

Brooks, R., Mather, J. S. B., \& Knowles, S. (2006). The influence of impact vibration modes and frequencies on cricket bat performance. Proceedings of the Institution of Mechanical Engineers, Part L: Journal of Materials Design and Applications, 220(4), 237-248.

Carré, M. J., James, D. M., \& Haake, S. J. (2004). Impact of a non-homogeneous sphere on a rigid surface. Proceedings of the Institution of Mechanical Engineers, Part C: Journal of Mechanical Engineering Science, 218(3), 273-281.

Draper, N. R., \& Smith, H. (1998). Applied regression analysis (3rd ed.). New York, NY: Wiley.

Elliott, B. C., Blanksby, B. A., \& Ellis, R. (1980). Vibration and rebound velocity characteristics of conventional and oversized tennis rackets. The Research Quarterly for Exercise and Sport, 51(4), 608-615.

Elliott, B. C. (1982). Tennis: the influence of grip tightness on reaction impulse and rebound velocity. Medicine and Science in Sports and Exercise, 14(5), 348-352.

Field, A. P. (2013). Discovering statistics using IBM SPSS statistics (4th ed.). London: Sage.

Justham, L., \& West, A. A. (2009). Design and development of a novel, integrated bowling machine for cricket. Proceedings of the Institution of Mechanical Engineers, Part P: Journal of Sports Engineering and Technology, 223(4), 125137.

King, M. A., Kentel, B. B., \& Mitchell, S. R. (2012). The effects of ball impact location and grip tightness on the arm, racquet and ball for one-handed tennis backhand groundstrokes. Journal of Biomechanics, 45(6), 1048-1052.

Knudson, D. (1993). Effect of String Tension and Impact Location on Ball Rebound Accuracy in Static Tennis Impacts. Journal of Applied Biomechanics, 9, 143-148

Mclntyre, D. R. \& Pfautsch, E. W. (1982). A kinematic analysis of the baseball batting swings involved in opposite-field and same-field hitting. Research Quarterly for Exercise and Sport, 53(3), 206-213.

Menard, S. (1995). Applied logistic regression analysis: Sage university series on quantitative applications in the social sciences. Thousand Oaks, CA: Sage.

Myers, R. (1990). Classical and modern regression with applications (2nd ed.). Boston, MA: Duxbury.

Nathan, A. M. (2003). Characterizing the performance of baseball bats. American Journal of Physics, 71(2), 134-143.

Noble, L. \& Eggeman, G. W. (1982). Baseball bat instrumentation for the measurement of hand forces. Journal of Biomechanics, 15(4), 341.

Peploe, C., King, M. A., \& Harland, A. R. (2014). The effects of different delivery methods on the movement kinematics of elite cricket batsmen in repeated front foot drives. Procedia Engineering, 72, 220-225.

Peploe, C., McErlain-Naylor, S. A., Harland, A. R., Yeadon, M. R., King, M. A. (2017). A curve fitting methodology to determine impact location, timing, and instantaneous post-impact ball velocity in cricket batting. Proceedings of the 
Institution of Mechanical Engineers, Part P: Journal of Sports Engineering and Technology. Advance online publication. doi: 10.1177/1754337117723275.

Shibayama, H. \& Ebashi, H. (1983). Development of a motor skill using the golf swing from the viewpoint of the regulation of muscle activity. Biomechanics VIII, 1, 895-902

Smith, L. (2001). Evaluating baseball bat performance. Sports Engineering, 4(4), 205-214

Stretch, R. A., Buys, F. J. \& Viljoen, G. (1995). The kinetics of the drive off the front foot in cricket batting: hand grip force. The South African Journal for Research in Sport, 18(2), 83-93.

Symes, A. W. (2006). The effect of mass distribution on cricket bat playing properties. (Unpublished PhD thesis). Loughborough University, Loughborough.

Weyrich, A. S., Messier, S. P., Ruhmann, B. S., \& Berry, M. J. (1989). Effects of bat composition, grip firmness, and impact location on post-impact ball velocity. Medicine and Science in Sports and Exercise, 21(2), 199-205.

Wherry, R. J. (1931). A new formula for predicting the shrinkage of the coefficient of multiple correlation. The Annals of Mathematical Statistics, 2(4), 440-457.

Worthington, P. J., King, M. A., \& Ranson, C. A. (2013). Relationships between fast bowling technique and ball release speed in cricket. Journal of Applied Biomechanics, 29(1), 78-84. 